ORAL PRESENTATIONS

June '93, Minneapolis, MN

\section{System Implications of the Ambulance Arrival to Patient Contact Interval on Response Time Compliance}

\author{
Campbell JP, ${ }^{*+}$ Gratton $M C$, ${ }^{*+}$ \\ Salomone JA, * Lindholm DJ, ${ }^{+}$Watson $\mathrm{WA}^{+* *}$ \\ * School of Medicine, University of Missouri-Kansas City, \\ Truman Medical Center \\ + Department of Health, City of Kansas City \\ ** School of Pharmacy, University of Missouri-Kansas City \\ Kansas City, Missouri, USA
}

Background: In some EMS system designs, response times are mandated with monetary penalties for non-compliance. These times are set with the goal of providing rapid, definitive patient care. The time interval between ambulance arrival and patient contact (APC) has been measured in EMS systems, but its effect on response time compliance has not been determined.

Purpose: To determine the effect of the APC interval on the mandated Code 1, $8 \mathrm{~min} 59 \mathrm{sec}$ and Code 2, $12 \mathrm{~min} 59 \mathrm{sec}$ response time compliance in an urban, public utility model system.

Methods: A prospective, observational study used independent, third-party riders to collect the APC interval for emergency lifethreatening (Code 1) and emergency non-life-threatening (Code 2) calls. The APC interval was added to the computeraided dispatch (CAD) time intervals to obtain the total time interval from call received until paramedic arrival at the patient's side. Compliance with the mandated response time was determined using $\mathrm{CAD}$ time intervals and $\mathrm{CAD}+\mathrm{APC}$ time intervals. Chi-square method was utilized to determine statistical significance.

Results: 198 of the 216 observed calls were matched to the CAD time intervals. 63 were Code 1 and 135 were Code 2 calls. 90.5\% of the Code 1 calls were compliant using CAD intervals with compliance dropping to $63.5 \%$ using CAD $+\mathrm{APC}(p<.0005)$. $94.1 \%$ of the Code 2 calls were compliant using CAD intervals with compliance decreasing to $83.7 \%$ using $\mathrm{CAD}+\mathrm{APC}(p=$ .012).

Conclusion: The CAD+APC intervals significantly impact system response time compliance. This must be considered when response time compliances are defined and mandated.

\section{EMS Transportation Decreases Time to Diagnosis and Treatment of Acute MI in the Emergency Department}

\author{
Swor $R$, Anderson $W{ }^{* *}$ Jackson $R$, Wilson $A^{*}$ \\ * Department of Emergency Medicine, \\ William Beaumont Hospital \\ Royal Oak, Michigan, USA \\ ** Department of Emergency Medicine, \\ William Beaumont Hospital \\ Troy, Michigan, USA
}

Introduction: Recent EMS literature has documented decreased time to emergency department thrombolytic therapy with the use of prehospital electrocardiography (ECG).

Purpose: Is the time to Emergency Department (ED) diagnosis and treatment of acute myocardial infarction (AMI) patients with thrombolytic agents decreased by EMS transport when compared to those transported by other methods (non-EMS).

Design: Retrospective, case control.

Study Population: AMI patients treated with thrombolytic agents at a community hospital ED with 34,000 visits during 1992.

Methods: Review of records of patients who received thrombolytic therapy for AMI. Statistical analysis was performed utilizing student t-test, Yates corrected Chi-square, and multiple linear regression.

Results: 87 patients received thrombolytic agents for AMI during 1992. Thirty-three arrived by ambulance, 54 arrived by other methods. There were no differences in age, gender, or time of ED arrival between these groups. Ambulance patients received standard advanced life support care, but not 12-lead ECG or thrombolytic agents. Ambulance patients experienced a significantly shorter time to first ECG (12.9+9.1 min vs 20.8 $\pm 25.3 \mathrm{~min})(p=.028)$, and received thrombolytic therapy sooner than did the controls $(56.0 \pm 31.5 \mathrm{~min}$ vs $78.0 \pm 63.4 \mathrm{~min})$ $(p=.018)$. This relationship persisted when analyzed controlling for age, gender, and shift $(\mathrm{F}=9.729, p=.003)$. There was no difference in time from diagnosis to treatment between these groups.

Conclusion: EMS transport of AMI patients in this study decreased time to diagnosis and treatment, and may be a confounder in studies that assess the value of field EMS interventions. Non-EMS AMI patients did not receive as rapid diagnosis and treatment, and emergency physicians should evaluate and address this issue in their departments. 